\title{
Development of Portable Fluorescence Microplate Reader Equipped with Indium Tin Oxide Glass Heater for Loop-mediated Isothermal Amplification
}

\author{
Ryo Ishii, ${ }^{1}$ Kazuhiro Morioka, ${ }^{2 *}$ Takuya Mizumoto, ${ }^{1}$ Natsumi Yamasaki, ${ }^{1}$ \\ Akihide Hemmi, ${ }^{3}$ Atsushi Shoji, ${ }^{2}$ Hiroya Murakami, ${ }^{4}$ Norio Teshima, ${ }^{4}$ \\ Tomonari Umemura, ${ }^{5}$ Katsumi Uchiyama, ${ }^{1}$ and Hizuru Nakajima ${ }^{1 * *}$ \\ ${ }^{1}$ Department of Applied Chemistry, Graduate School of Urban Environmental Sciences, \\ Tokyo Metropolitan University, 1-1 Minami-ohsawa, Hachioji, Tokyo 192-0397, Japan \\ ${ }^{2}$ Department of Biomedical Analysis, School of Pharmacy, \\ Tokyo University of Pharmacy and Life Sciences, 1432-1 Horinouchi, Hachioji, Tokyo 192-0392, Japan \\ ${ }^{3}$ Mebius Advanced Technology Ltd., 3-31-6 Nishiogi-kita, Suginami-ku, Tokyo 167-0042, Japan \\ ${ }^{4}$ Department of Applied Chemistry, Aichi Institute of Technology, \\ 1247 Yachigusa, Yakusa-cho, Toyota, Aichi 470-0392, Japan \\ ${ }^{5}$ School of Life Sciences, Tokyo University of Pharmacy and Life Sciences, \\ 1432-1, Horinouchi, Hachioji, Tokyo 192-0392, Japan
}

(Received September 3, 2021; accepted November 18, 2021; online published January 5, 2022)

Keywords: fluorescence microplate reader, LAMP, ITO glass heater, on-site nucleic acid test, variety discrimination

We developed a portable fluorescence microplate reader equipped with an indium tin oxide (ITO) glass heater for nucleic acid testing based on loop-mediated isothermal amplification (LAMP). The components used for detection (e.g., LEDs, optical filters, the microwell plate, the ITO glass heater, and photodiodes) are arranged in parallel on a straight line. This optical placement allows us to simultaneously heat nine sample solutions and monitor the fluorescence intensity amplified by the LAMP reaction despite the analysis instrument being handheld. This simultaneous monitoring was demonstrated by the LAMP reaction using positive and negative controls. In addition, the microplate reader was applied to the variety discrimination of rice, and Koshihikari rice was successfully discriminated from four types of milled rice using the microplate reader. The developed microplate reader will be useful for on-site nucleic acid testing because it can be operated using batteries and is portable.

\section{Introduction}

Recently, portable nucleic acid test systems for on-site diagnosis have attracted a lot of attention. ${ }^{(1-3)}$ Carrying out nucleic acid testing in the field is useful for point-of-care testing, ${ }^{(4)}$ environmental analysis, ${ }^{(5)}$ and food safety testing ${ }^{(6)}$ because the result can be obtained rapidly. To miniaturize and reduce the cost of nucleic acid test systems, the design of the detection and heating parts is very important. Although the real-time polymerase chain reaction $(\mathrm{PCR})^{(7)}$ is the

\footnotetext{
*Corresponding author: e-mail: kmorioka@toyaku.ac.jp

** Corresponding author: e-mail: nakajima-hizuru@tmu.ac.jp

https://doi.org/10.18494/SAM.2022.3618
} 
gold standard method among nucleic acid amplification techniques, the analysis requires a device for multistep heating and a highly sensitive detection system for measuring the fluorescence signal of dye molecules existing at low concentrations. Various types of miniature real-time PCR systems have been developed, ${ }^{(8-10)}$ but the systems are large and complicated in many cases. In addition, the real-time PCR requires a long analysis time for the heater control cycle as well as for PCR amplification.

Loop-mediated isothermal amplification (LAMP), ${ }^{(11)}$ which is one of the nucleic acid amplification methods, is very useful for on-site nucleic acid testing since it is capable of rapid nucleic acid amplification by isothermal heating. LAMP can be adapted to various detection methods [e.g., turbidimetry ${ }^{(12)}$ and colorimetry ${ }^{(13)}$ ] as well as to the fluorescence detection method, ${ }^{(14,15)}$ and LAMP enables us to discriminate the presence or absence of gene amplification visually. ${ }^{(16-18)}$ Moreover, isothermal heating enables us to simplify the system configuration. Owing to these advantages, many research groups have studied LAMP systems based on visual detection for on-site nucleic acid testing. ${ }^{(19-21)}$ Although visual discrimination is simple and does not require a detection device, the obtained result is semi-quantitative. On the other hand, real-time signal monitoring during the LAMP reaction ${ }^{(22)}$ is useful for rapidly finding abnormalities caused by contamination as well as for the quantitation of nucleic acid. More recently, some detection devices have been developed for real-time LAMP reaction monitoring, such as a small detection system using an LED and a photodiode (PD), ${ }^{(22,23)}$ a tube scanner, ${ }^{(24)}$ and a smartphone-based detection system. ${ }^{(25)}$ These detection devices were not only small but also easy to use and had a high detection sensitivity and a low fabrication cost. On the other hand, for the heating part of the LAMP system, downsizing, weight saving, low electric power consumption, and temperature stability are required. Various types of heaters [e.g., film heaters $^{(26,27)}$ and heating coils $\left.{ }^{(28)}\right]$ have been used for portable LAMP systems. The performance of these heaters is sufficient to perform the LAMP reaction, but the optical arrangement of the detection system is limited because of the opaque heater material.

An indium tin oxide (ITO) glass heater ${ }^{(29)}$ has advantages such as easy fabrication at a low cost and heating with low electrical power, similarly to other heaters used for portable analysis systems. Moreover, several ITO heaters can be fabricated on a single glass plate by chemical etching. ${ }^{(30-32)}$ However, the most noteworthy feature of ITO heaters is the high optical transmittance. Since an ITO heater can transmit light in the visible and near-IR regions, it is useful for measuring a heated substance using optical detection methods. Because of these characteristics, ITO heaters have been widely used in biological analyses such as cell analysis ${ }^{(33)}$ and hybridization. ${ }^{(34)}$ Several research groups have applied an ITO heater to gene testing based on PCR. ${ }^{(30-32)} \mathrm{Wu}$ et al. developed a thermostat chip with an ITO glass substrate for static PCR. ${ }^{(35)}$ The fabricated system including the thermostat chip was successfully applied to the in situ real-time monitoring of the fluorescence of SYBR Green I during PCR. However, it is difficult to apply the system to on-site gene testing because it requires large and expensive peripheral instruments, i.e., a fluorescence microscope and a photomultiplier for fluorescence detection. Until now, an ITO glass heater has not been applied to a portable simultaneous gene testing device for the real-time fluorescence monitoring of the LAMP reaction. The main reason for this is that a fluorescence detector commonly requires an optical system, in which a light 
source and a photodetector are vertically arranged, increasing the size and complexity of the detection system.

Previously, we developed a portable fluorescence microplate reader for enzyme-linked immunosorbent assay (ELISA). ${ }^{(36)}$ The optical components of the microplate reader, i.e., light sources, two optical filters, and photodetectors, were arranged in parallel on a straight line. This optical system enabled us to detect the fluorescence signal in nine microwells simultaneously. The microplate reader was successfully applied to detect immunoglobulin A (IgA) in human saliva.

Herein, we developed a portable fluorescence microplate reader equipped with an ITO glass heater for LAMP. The flat heater with high transparency and the detection components were arranged in parallel to monitor fluorescence intensity changes in nine wells during the LAMP reaction simultaneously. The heating and detection performance characteristics of the microplate reader were precisely evaluated and then the microplate reader was applied to the variety discrimination of rice based on LAMP.

\section{Materials and Methods}

\subsection{Reagents and materials}

Water purified by a Direct-Q system (Nihon Millipore, Tokyo, Japan) was used for sample preparation. All chemical reagents used in this work were of analytical grade unless otherwise stated. The polydimethylsiloxane (PDMS) base compound and curing reagent (DOWSIL ${ }^{\mathrm{TM}}$ SILPOT $184 \mathrm{~W} / \mathrm{C}$ ) were purchased from Dow Corning Toray Co., Ltd. (Tokyo, Japan). Carbon black (K-COLOR-BK-02) was purchased from Shin-Etsu Chemical Co., Ltd. (Tokyo, Japan). Ag/ $\mathrm{AgCl}$ paste was purchased from Gwent Group Co., Ltd. (Gwent, UK). Calcein and hydrochloric acid were purchased from Kanto Chemical Co., Ltd. (Tokyo, Japan). 2-Amino-2-hydroxymethyl1,3-propanediol (Tris) was purchased from Fujifilm Wako Pure Chemical Co., Ltd. (Osaka, Japan). Chlorine bleach was purchased from Kao Co., Ltd. (Tokyo, Japan). The Koshihikari LAMP discrimination kit containing a LAMP primer, an enzyme reagent, a fluorescence color reagent, positive and negative controls, and sterilized water was purchased from Nippon Gene Co., Ltd. (Tokyo, Japan).

\subsection{Fabrication of nine-well microtiter plate}

The mold of the nine-well microtiter plate was fabricated using polyoxymethylene (POM) plates and cylindrical stainless pins ( $(4.0 \mathrm{~mm} \times \mathrm{H} 10 \mathrm{~mm})$. A baseplate (L $50 \mathrm{~mm} \times \mathrm{W} 50 \mathrm{~mm} \times$ $\mathrm{H} 10 \mathrm{~mm}$ ) having nine holes $(\varphi 4.0 \mathrm{~mm} \times \mathrm{D} 5 \mathrm{~mm})$ and a square convex portion (L $30 \mathrm{~mm} \times \mathrm{W}$ $30 \mathrm{~mm} \times \mathrm{H} 3 \mathrm{~mm}$ ), and a mold form (L $50 \mathrm{~mm} \times \mathrm{W} 50 \mathrm{~mm} \times \mathrm{H} 8 \mathrm{~mm}$ ) having a square through hole (L $30 \mathrm{~mm} \times \mathrm{W} 30 \mathrm{~mm}$ ) were formed using a milling machine (KitMill RD420, ORIGINALMIND Co., Ltd., Nagano, Japan). The cylindrical stainless pins were inserted into each hole of the baseplate after the bottom surface of the pins was polished with a liquid abrasive (Pikal, Nihon Maryo Kogyo Co., Ltd., Tokyo, Japan). The mold of the nine-well microtiter plate 
was fabricated by fitting the mold form into the baseplate and then fixed by taping the outside of the plates with an adhesive tape.

The nine-well microtiter plate was made from PDMS and carbon black paste. The fabrication procedure for the nine-well microtiter plate is shown in Fig. 1(a). The PDMS base compound and curing agent were mixed at a weight ratio of 10 to 1 . The PDMS mixture and carbon black paste were mixed at a weight ratio of 19 to 1 , and this black mixture was degassed using a vacuum pump (G-25SA, ULVAC, Inc., Kanagawa, Japan). The degassed black mixture was poured into the mold and the mold was covered with an acrylic block $\left(50 \times 50 \times 50 \mathrm{~mm}^{3}\right.$, Mebius Advanced Technology Ltd., Tokyo, Japan). The mold containing the black mixture was heated at $70{ }^{\circ} \mathrm{C}$ for $1.5 \mathrm{~h}$ in an oven (ST100, ESPEC Co., Ltd., Osaka, Japan) to polymerize the PDMS. After that, the cured black PDMS plate was peeled off from the mold, and nine through holes $(\varphi 3.5 \mathrm{~mm})$ were formed on the black PDMS plate using a biopsy punch (BP-35F, Kai Industries Co., Ltd., Tokyo, Japan). This plate was returned to the mold and then the PDMS mixture without carbon black was poured on the mold. The mold was covered with the acrylic block and then cured at 70 ${ }^{\circ} \mathrm{C}$ for $1.5 \mathrm{~h}$. After the PDMS was cured, the finished nine-well microtiter plate was peeled off from the mold. The design and photograph of the nine-well microtiter plate are shown in Figs. 1(b) and 2(b), respectively. Since it was difficult to completely remove amplicon by washing the wells, the nine-well microtiter plate was not reused. Before use, the microtiter plate was washed with $1.0 \%(\mathrm{v} / \mathrm{v})$ chlorine bleach aqueous solution to prevent contamination.

\subsection{Fabrication of ITO transparent heater}

A piece of ITO transparent glass $\left(100 \times 100 \times 1.1 \mathrm{~mm}^{3}\right.$, No. 1001, Geomatec Co., Ltd., Tokyo, Japan) was cut to $20 \times 40 \mathrm{~mm}^{2}$ using a glass cutter, then cleaned with ultrapure water. After drying, two wires were fixed on both ends of the ITO glass with $\mathrm{Ag} / \mathrm{AgCl}$ paste, and the paste was cured at $150{ }^{\circ} \mathrm{C}$ for $30 \mathrm{~min}$. The performance of the ITO glass heater was evaluated using a thermal camera (PI400i, Argo Co., Ltd., Berlin, Germany).

(a)

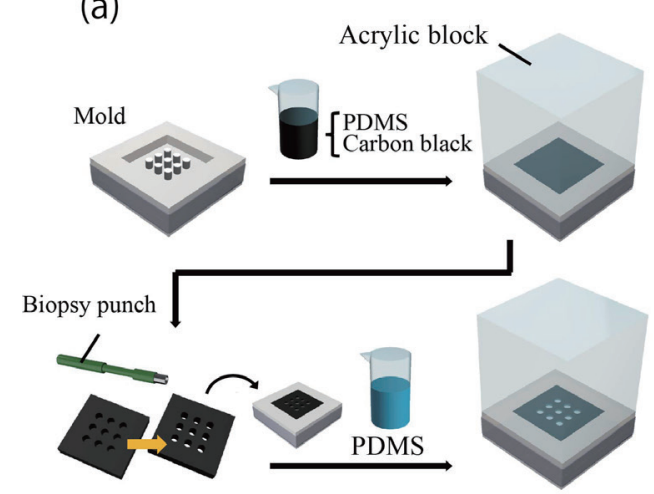

(b)

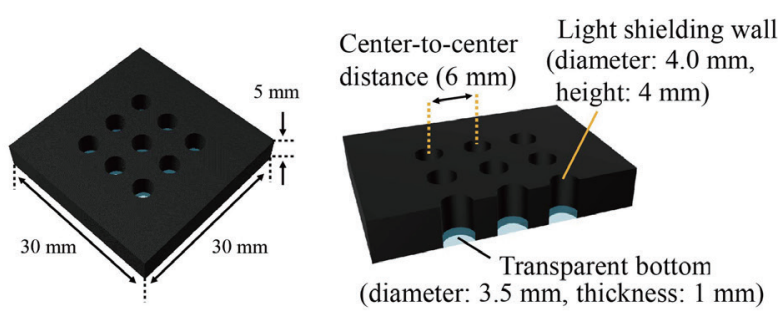

Fig. 1. (Color online) Nine-well microtiter plate made from PDMS and carbon black. (a) Fabrication procedure for Xthe plate. (b) Design of the plate. 


\subsection{Development of portable fluorescence microplate reader}

The optical design and photograph of the portable fluorescence microplate reader developed in this work are shown in Fig. 2. The optical system of the plate reader consisted of nine highbrightness blue LEDs (OSUB5161P, OptoSupply Ltd., N.T., Hong Kong), a short-pass filter (SPF) (SVX490, Asahi Spectra Co., Ltd., Tokyo, Japan), a long-pass filter (LPF) (LVX530, Asahi Spectra), the ITO glass heater, two perforated light-shielding plates, and nine PDs (TEMD7000X01, Vishay Intertechnology, Inc., PA, USA). The detection of isothermal gene amplification using the microplate reader was performed as follows. The nine-well microtiter plate was set in the plate reader and the sample solutions in the nine microwells on the microtiter plate were heated using the ITO glass heater until the measurement was finished. The excitation light beams from the LEDs (peak emission wavelength: $467 \mathrm{~nm}$ ) were passed through the SPF (cut-off wavelength: $490 \mathrm{~nm}$ ) and ITO glass heater, and then irradiated the solutions in the microwells. The fluorescence of calcein generated in the microwells was passed through the LPF (cut-on wavelength: $530 \mathrm{~nm}$ ) and reached the PDs. The fluorescence was converted to photocurrent by the PDs. The photocurrent was converted to an analog voltage and amplified by a current/voltage (I/V) conversion amplifier (Mebius Advanced Technology Ltd., Tokyo, Japan). The voltage was converted to a digital value by an analog/digital (A/D) converter (ADS1115, Adafruit Industries, NY, USA). The digital values were collected by a central processing unit (CPU) (Arduino Nano 3.0, Gravitech LLC, NV, USA) as measurement data once per second, and the data were transmitted to a laptop PC by a Bluetooth communication module [Bluefruit EZLink - Bluetooth Serial Link \& Arduino Programmer (v 1.3), Adafruit Industries]. The temperature of the ITO glass surface was monitored with a thermistor (103 JT-025, SEMITEC

(a)

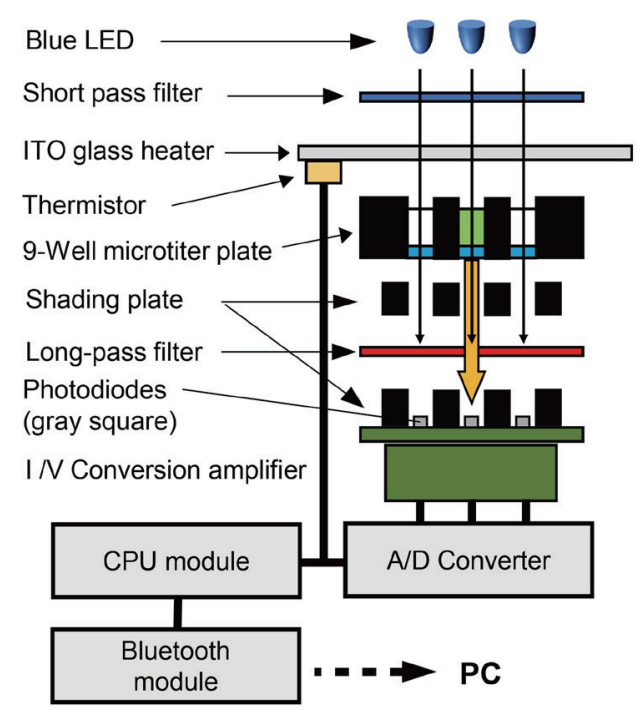

(b)

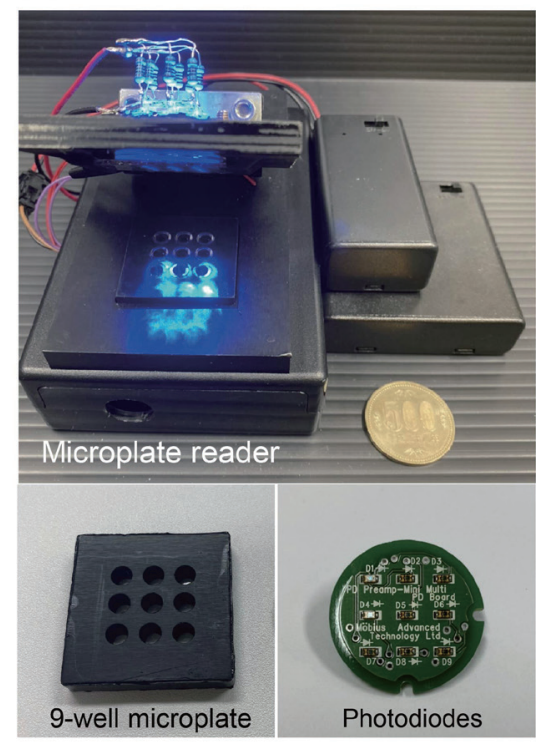

Fig. 2. (Color online) Portable microplate reader developed in this work. (a) Optical design of the microplate reader. (b) Photographs of the microplate reader, nine-well microtiter plate, and photodiodes. 
Co., Ltd., Tokyo, Japan) and heating was controlled using the CPU to a constant temperature by setting the heater to turn on when the temperature was below $63{ }^{\circ} \mathrm{C}$. In a result obtained by measuring a positive control, the fluorescence increases with time because the amount of the target gene is increased by LAMP. The current applied to the LEDs was set to ca. $20 \mathrm{~mA}$. The plate reader was driven using four $1.2 \mathrm{~V}$ batteries (Toshiba Co., Ltd., Tokyo, Japan) and a DC power supply (Sunhayato Co., Ltd., Tokyo, Japan). The total size and weight of the plate reader were $130 \mathrm{~mm}(\mathrm{~L}) \times 80 \mathrm{~mm}(\mathrm{~W}) \times 80 \mathrm{~mm}(\mathrm{H})$ and approximately $400 \mathrm{~g}$, respectively.

\subsection{LAMP reaction}

The variety discrimination of rice was carried out using the LAMP kit. Using this kit, Koshihikari rice is identified by discriminating the presence or absence of the amplification of the region that corresponds to the first intron of resistance gene Pi5-1. Gene testing using the LAMP kit enables Koshihikari to be distinguished from other breeds. In this experiment, four types of milled rice (Koshihikari, Akitakomachi, Hitomebore, and Yumepirika) were used as samples. DNA extraction was performed according to the attached manual of the LAMP kit. ${ }^{(37)}$ A $10 \mathrm{~g}$ sample of milled rice was placed in a centrifuge tube and then $19 \mathrm{~mL}$ of sterilized water and $1 \mathrm{~mL}$ of $20 \times$ DNA extraction solution No. 1 were added to the tube. After mixing, $500 \mu \mathrm{L}$ of the supernatant in the tube was transferred to a microtube and then an equal volume of DNA extraction solution No. 2 was added to the microtube. The solution was mixed and then centrifuged at $12000 \mathrm{~g}$ for 1 min using a centrifuge (HSC-12000, AS ONE Co., Ltd., Osaka, Japan). The supernatant in the tube was collected in another tube and the solution was diluted tenfold with sterilized water. The prepared solution was used as a sample solution in the following experiment.

The LAMP reaction in this work was performed using the Koshihikari LAMP discrimination kit and a fluorescent detection reagent containing calcein and manganese. Twenty-five microliters of a solution comprising $20.5 \mu \mathrm{L}$ of the test reagent (No. 1 or No. 2), $1 \mu \mathrm{L}$ of the fluorescence detection reagent, $1 \mu \mathrm{L}$ of an enzyme solution, and $2.5 \mu \mathrm{L}$ of the sample solution was added to a microwell on the microtiter plate. The microtiter plate was set in the developed microplate reader and then the amplification was carried out at $63{ }^{\circ} \mathrm{C}$ for $40 \mathrm{~min}$. During the LAMP reaction, the fluorescence intensity of the solution in the microwell was monitored in real time. The breeds of rice were discriminated by comparing the fluorescence intensity of the sample solution with those of the positive and negative controls.

\section{Results and Discussion}

\subsection{Optical design of fluorescence microplate reader}

The fluorescence reagent for LAMP contains calcein. The fluorescence of calcein in the reagent is initially quenched by binding with manganese ions. As the isothermal amplification reaction proceeds, the generated pyrophosphate removes the manganese ions from calcein, and accordingly the calcein fluoresces. The fluorescence of calcein increases in the presence of 


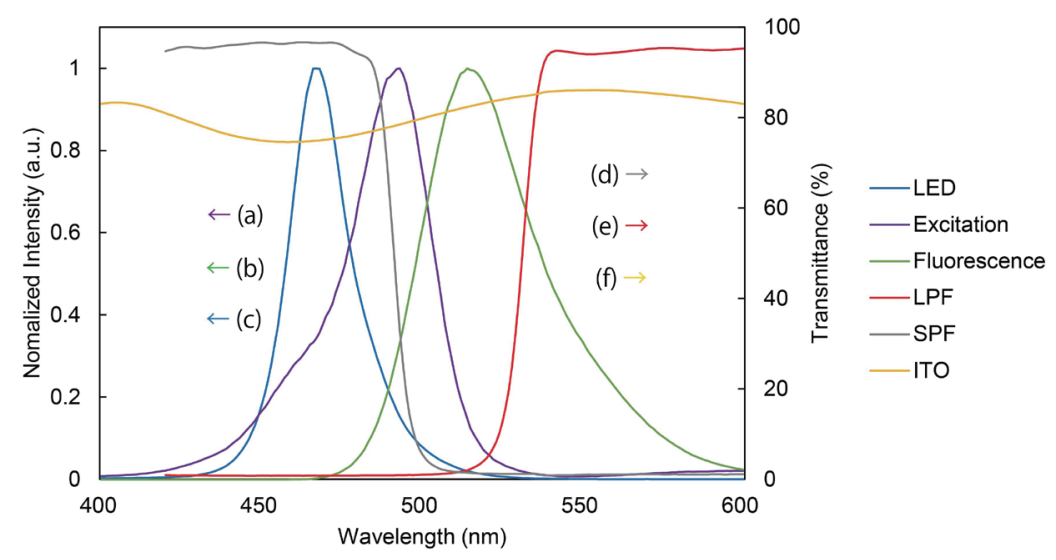

Fig. 3. (Color online) Optical spectra of the reagent and parts of the microplate reader. (a) Excitation spectrum of calcein. (b) Emission spectrum of calcein. (c) Excitation spectrum of the blue LED. (d) Transmission spectrum of the SPF. (e) Transmission spectrum of the LPF. (f) Transmission spectrum of the ITO glass heater. Arrows next to the letters show the corresponding axis.

manganese ions in the reaction mixture. On the basis of this principle, we designed the optical system of the fluorescence microplate reader for detecting the fluorescence of calcein. The optical spectra of calcein were investigated using a spectrofluorometer (RF-5300PC, Shimadzu Co., Ltd., Kyoto, Japan) [Figs. 3(a) and 3(b)]. The wavelengths of maximum excitation and emission were 493 and $512 \mathrm{~nm}$, respectively. In the developed microplate reader, the increase in background signal intensity due to excitation light was a concern because the LEDs and PDs were arranged in parallel on a straight line to detect fluorescence in nine microwells simultaneously.

To overcome this problem, high-brightness blue LEDs having a peak emission wavelength of less than $512 \mathrm{~nm}$ were selected as an excitation light source, and the SPF and LPF were mounted between the LEDs and the PDs. The emission spectrum of the LED was measured using a spectrometer (Colorcompass MF, AT System, Shizuoka, Japan). The peak emission wavelength and full width at half maximum of the LED were 467 and $22 \mathrm{~nm}$, respectively [Fig. 3(c)]. Figures 3(d) and 3(e) show the transmission spectra of the filters. The cut-off wavelength of the SPF and the cut-on wavelength of the LPF were 490 and $530 \mathrm{~nm}$, respectively. The microplate reader with the filters successfully detected the fluorescence of calcein without a background signal. The transmittance property of the ITO glass heater was investigated, and the transmittance at 467 $\mathrm{nm}$ (i.e., the peak emission wavelength of the LED) was approximately $75 \%$ [Fig. 3(f)]. The result revealed that the fluorescence of calcein can be detected using the plate reader equipped with the ITO heater without any problems.

\subsection{Evaluation of optical crosstalk}

To prevent the generation of background noise caused by stray light, the nine-well microtiter plate was fabricated from the PDMS mixture containing carbon black, except for the bottom of the microwells. Although the opacity of the black PDMS plate containing carbon black was 
sufficient, optical crosstalk might have occurred because of the short distance between the microwells $(6 \mathrm{~mm})$ and because the fluorescence in the nine microwells was detected using nine LEDs and nine PDs arranged in close proximity. Thus, the effect of the crosstalk was evaluated using calcein solution. A $25 \mu \mathrm{L}$ portion of $50 \mu \mathrm{M}$ calcein solution prepared with $50 \mathrm{mM}$ Tris$\mathrm{HCl}$ buffer ( $\mathrm{pH}$ 8.0) (Tris buffer) was added to one of the microwells, whereas a $25 \mu \mathrm{L}$ portion of the Tris buffer was added to the other wells, and then the fluorescence intensities in all the microwells were measured using the developed microplate reader. Figure 4 shows the results of the crosstalk evaluation performed for all microwells in turn. Compared with the fluorescence intensity in the microwell in which calcein solution was added, the fluorescence intensities in the other microwells were very low. This result indicates that the two light-shielding plates with nine through holes arranged above and below the LPF prevented the incidence of fluorescence emitted from the microwell into the PDs corresponding to the other microwells, and the fluorescence in the nine microwells can be detected normally without crosstalk.

\subsection{Evaluation of quantitative performance}

To evaluate the quantitative performance of the developed microplate reader, calcein solutions at several concentrations were measured. A $25 \mu \mathrm{L}$ portion of a calcein solution diluted with the Tris buffer was added to the microwells, and the fluorescence intensities were measured using
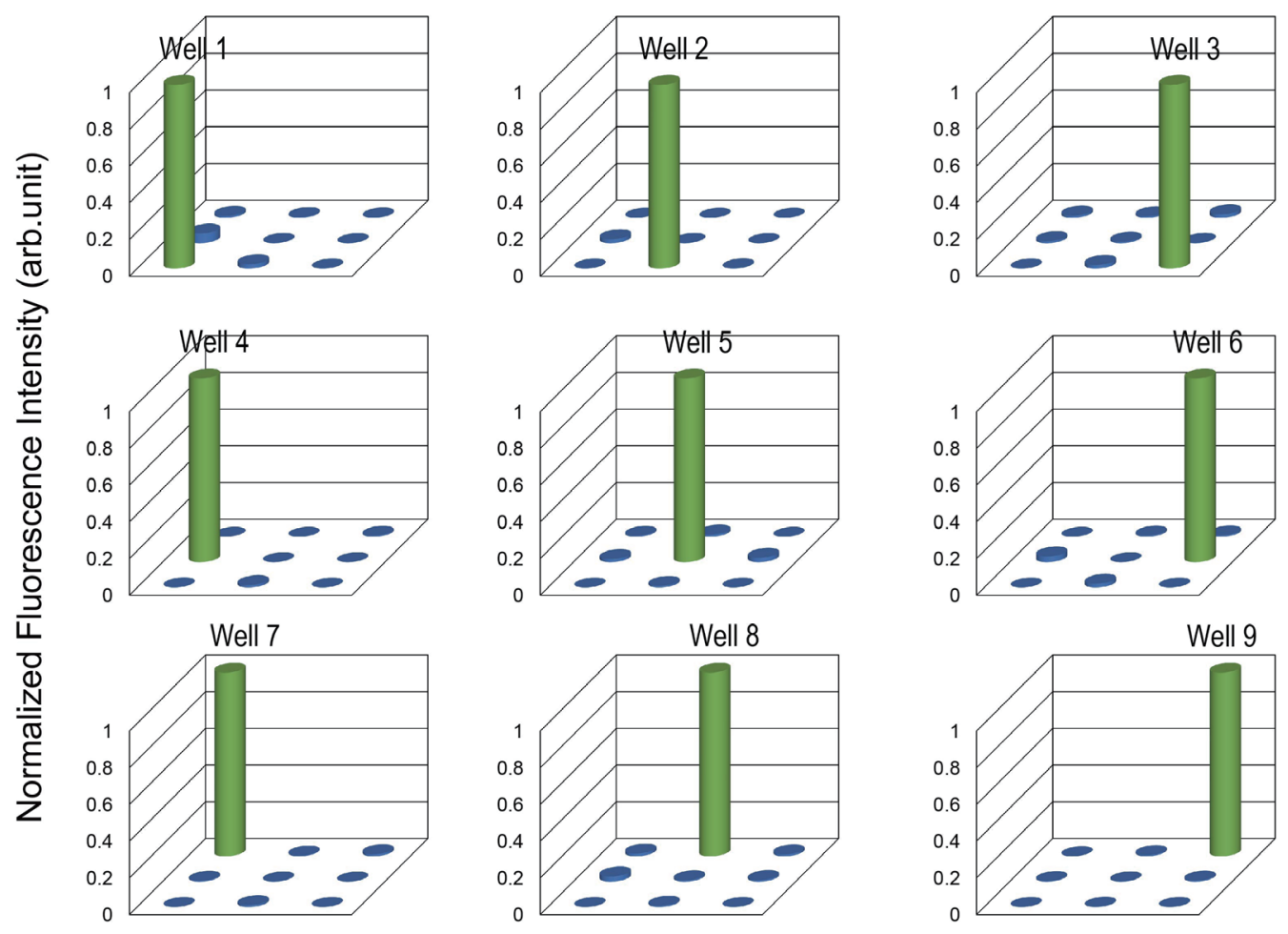

Fig. 4. (Color online) Fluorescence intensity in nine microwells when the solution containing calcein was added to each microwell and water was added to the other microwells. 

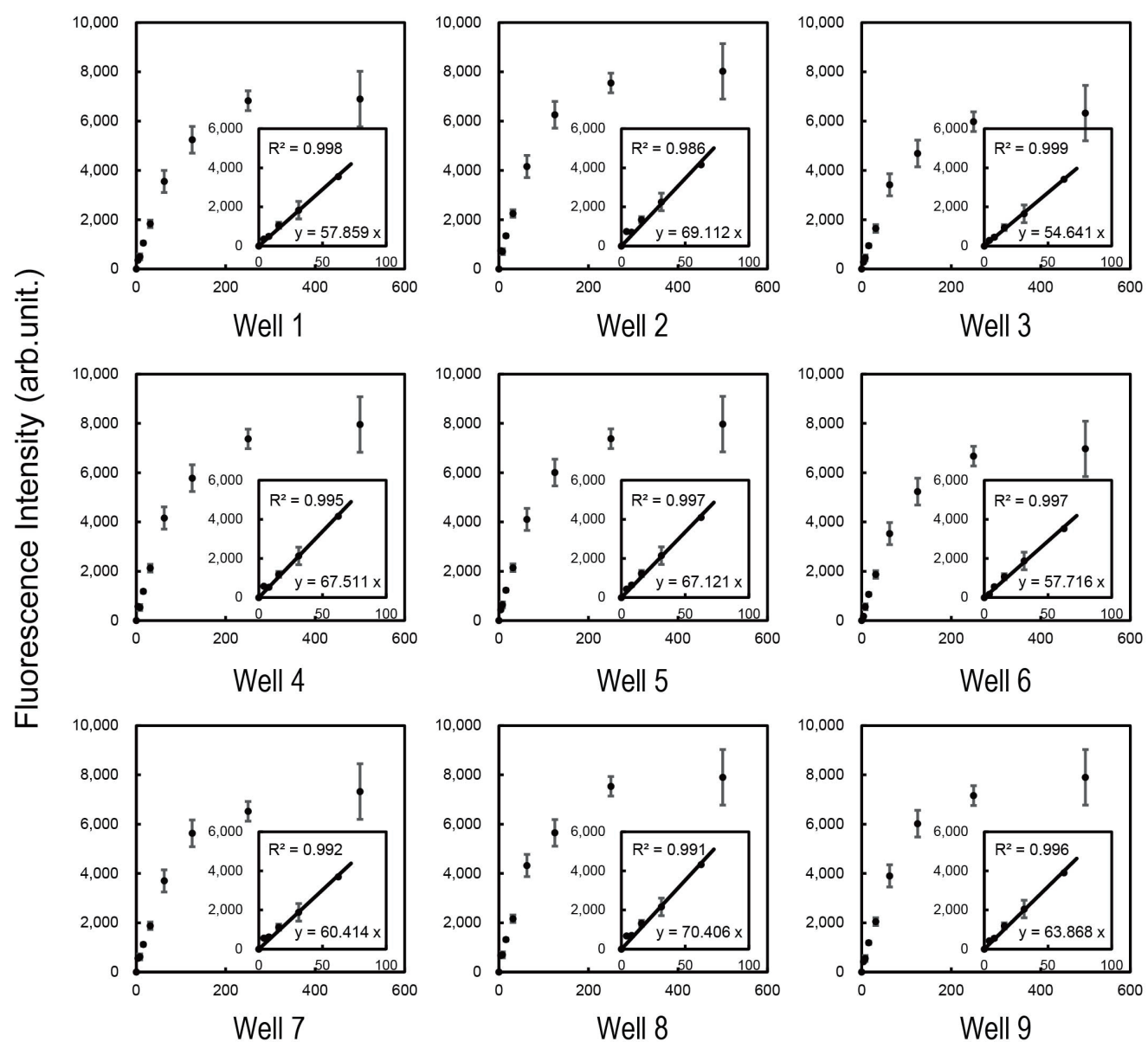

Well 2
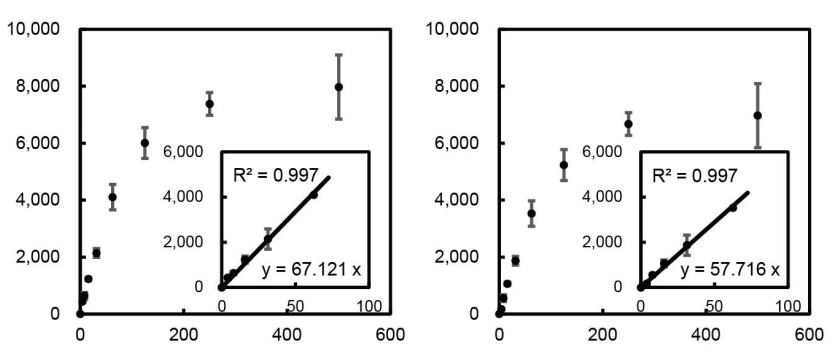

Well 5

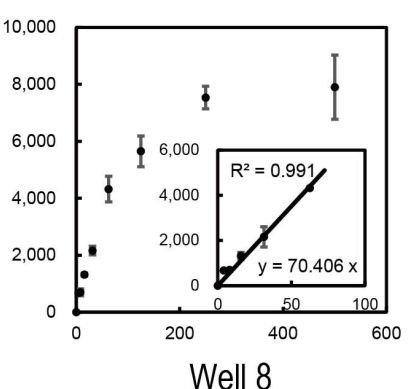

Well 6

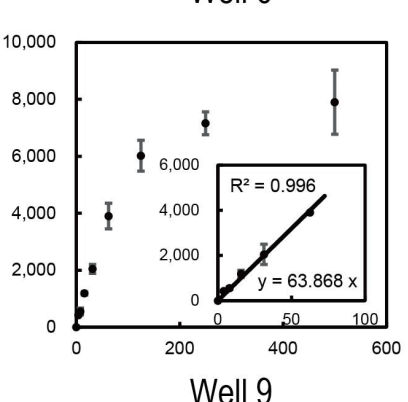

Concentration of Calcein $(\mu \mathrm{M})$

Fig. 5. Calibration curves for calcein.

the microplate reader. The obtained calibration curves for calcein are shown in Fig. 5. The fluorescence intensity increased with the concentration of calcein and became almost constant for concentrations above $250 \mu \mathrm{M}$. The calibration curves of each well for calcein were linear for concentrations below $63 \mu \mathrm{M}$ with correlation coefficients of $0.991-0.999$. The limits of detection (LODs), defined as three times the standard deviation of the blank signal, were estimated to be $0.27-1.60 \mu \mathrm{M}$. The relative standard deviations (RSDs) $(n=4)$ in the detectable range (i.e., the concentration range from the LOD to $63 \mu \mathrm{M}$ ) for each well were below $14.1 \%$. These results indicate that the fluorescence intensity of calcein solution can be measured quantitatively using the developed plate reader.

\subsection{Heating performance of ITO glass heater}

To simultaneously perform the LAMP reaction in all the microwells using the microplate reader, all microwells must be heated uniformly and stably to the temperature necessary for the isothermal amplification reaction using the ITO glass heater. Thus, the temperature uniformity 
(a)

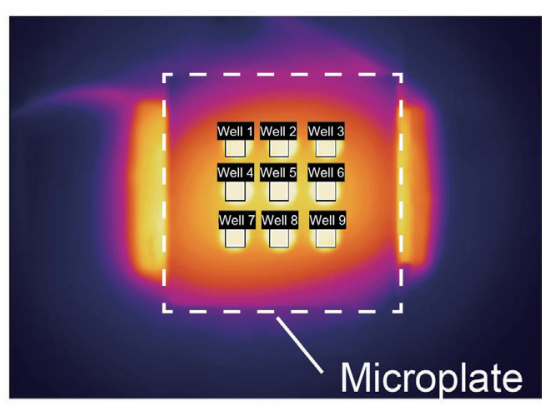

(b)

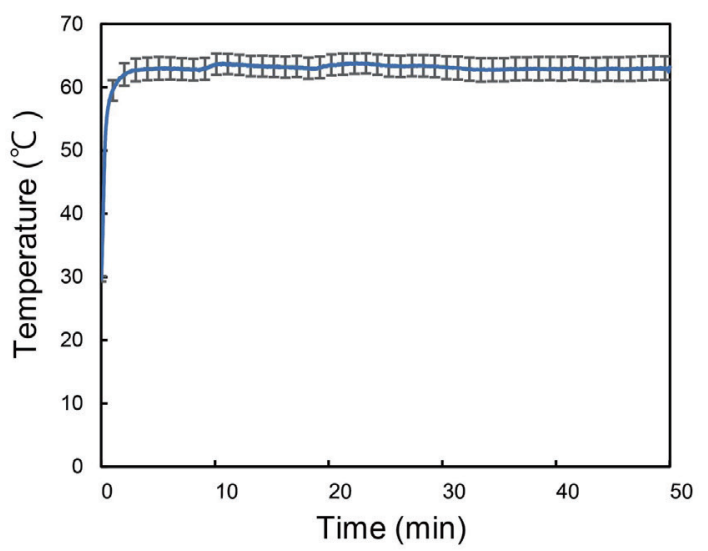

Fig. 6. (Color online) Heating performance of the ITO glass heater. (a) Thermal image of the microtiter plate heated by the ITO glass heater. (b) Time course of the temperature in the microwells. The line shows the average temperature measured in the nine microwells. Heating was carried out for $40 \mathrm{~min}$.

between the microwells was investigated by monitoring the temperatures of water in the nine microwells heated to $63{ }^{\circ} \mathrm{C}$ by the ITO glass heater using a thermal camera. The thermal camera was arranged on the opposite side from the ITO glass heater. The obtained thermogram [Fig. 6(a)] indicates that the ITO glass heater has sufficient performance to heat the microwells uniformly. From this data, the average temperature of water in the nine microwells was estimated to be $62.5 \pm 2.0^{\circ} \mathrm{C}$. This result suggests that solutions in all microwells were heated to almost the set temperature $\left(63{ }^{\circ} \mathrm{C}\right)$ and that the heating ability is sufficient for the LAMP reaction.

To carry out the LAMP reaction for the variety discrimination of rice using the plate reader, the sample solution containing the reagents required heating to $63{ }^{\circ} \mathrm{C}$ for $40 \mathrm{~min}$. Thus, the temporal temperature stability in the nine microwells was evaluated by measuring the temperatures of water in the microwells heated to $63{ }^{\circ} \mathrm{C}$. As shown in Fig. 6(b), the average temperature of water in the nine microwells reached $63{ }^{\circ} \mathrm{C}$ within $95 \mathrm{~s}$ from the start of heating, and the temperature remained almost constant for $40 \mathrm{~min}$. After reaching $63{ }^{\circ} \mathrm{C}$, the average temperature of water in the nine microwells for the next $40 \mathrm{~min}$ was calculated to be $60.4 \pm 0.4$ ${ }^{\circ} \mathrm{C}-65.4 \pm 0.2{ }^{\circ} \mathrm{C}$. In addition, the battery power provided to the heater and peripheral devices was sufficient for the continuous heating under this condition. These results revealed that the performance of the ITO glass heater was sufficient to perform the LAMP reaction.

\subsection{Variety discrimination of rice}

To demonstrate the variety discrimination of real samples extracted from milled rice, a LAMP test was performed using a thermal cycler and a UV illuminator. A total of 12 PCR tubes containing different breeds of rice, the positive control, or the negative control, as shown in Table 1 , were heated to $63{ }^{\circ} \mathrm{C}$ for 40 min using a commercially available thermal cycler (TCLT9610, Blue-Ray Biotech Co., Ltd., Zhongshan Dist., Taipei), and then the fluorescence of the 
Table 1

Analytes and reagents used for the variety discrimination of rice.

\begin{tabular}{rccc}
\hline Sample No. & Analyte & Reagent & Reaction \\
\hline 1 & \multirow{2}{*}{ Koshihikari } & No. 1 & $(+)$ \\
2 & & No. 2 & $(-)$ \\
\hline 3 & \multirow{2}{*}{ Akitakomachi } & No. 1 & $(-)$ \\
4 & No. 2 & $(+)$ \\
\hline 5 & \multirow{2}{*}{ Hirombore } & No. 1 & $(-)$ \\
6 & No. 2 & $(+)$ \\
\hline 7 & \multirow{2}{*}{ Yumepirika } & No. 1 & $(-)$ \\
8 & \multirow{2}{*}{ Positive control } & No. 2 & $(+)$ \\
\hline 9 & & No. 1 & $(+)$ \\
10 & \multirow{2}{*}{ Negative control } & No. 2 & $(+)$ \\
\hline 11 & & No. 2 & $(-)$ \\
12 & & & $(-)$ \\
\hline
\end{tabular}

solutions was visually observed by irradiating UV light to the solutions using a UV illuminator (SLUV- 4, AS ONE Co., Ltd., Osaka, Japan).

When test reagent No. 1 was used, the solutions containing the positive control or Koshihikari emitted fluorescence, whereas no fluorescence was observed in the solutions containing the negative control or the other types of rice. On the other hand, when test reagent No. 2 was used, the solutions containing the positive control or the rice except for Koshihikari emitted fluorescence, whereas no fluorescence was observed in the solutions containing the negative control or Koshihikari. These results were identical with those in the manual. ${ }^{(37)}$

To demonstrate that the developed microplate reader can be used for the LAMP reaction, the LAMP amplification of the positive and negative controls was carried out in the nine microwells using the developed microplate reader. The time course of the fluorescence intensities during the LAMP amplification is shown in Fig. 7. Specific differences in fluorescence intensity between the positive and negative controls were observed in all microwells. This result revealed that the developed microplate reader can be used to heat the solution in the microwells and that the fluorescence intensity in all microwells can be monitored in real time during the LAMP reaction using the developed microplate reader. The time until the fluorescence intensity started to increase rapidly was different in each microwell. A possible reason for this is that the temperature in each microwell and the sensitivity of each PD were slightly different. The performance of the developed microplate reader was sufficient to discriminate the positive and negative controls. However, for the simultaneous determination of DNA, the preparation of calibration curves for each microwell was required since the heating and detection performance characteristics were different in each microwell.

In the variety discrimination of rice, a decision of the presence or absence of a specific gene sequence is only required. Therefore, the performance of the developed microplate reader is sufficient for the variety discrimination of rice. The variety discrimination of rice was carried out using the developed microplate reader. The time courses of the fluorescence intensity in the microwells for four rice samples, the positive control, and the negative control are shown in Fig. 8(a). When test reagent No. 1 was used, the fluorescence intensity of the solution containing 

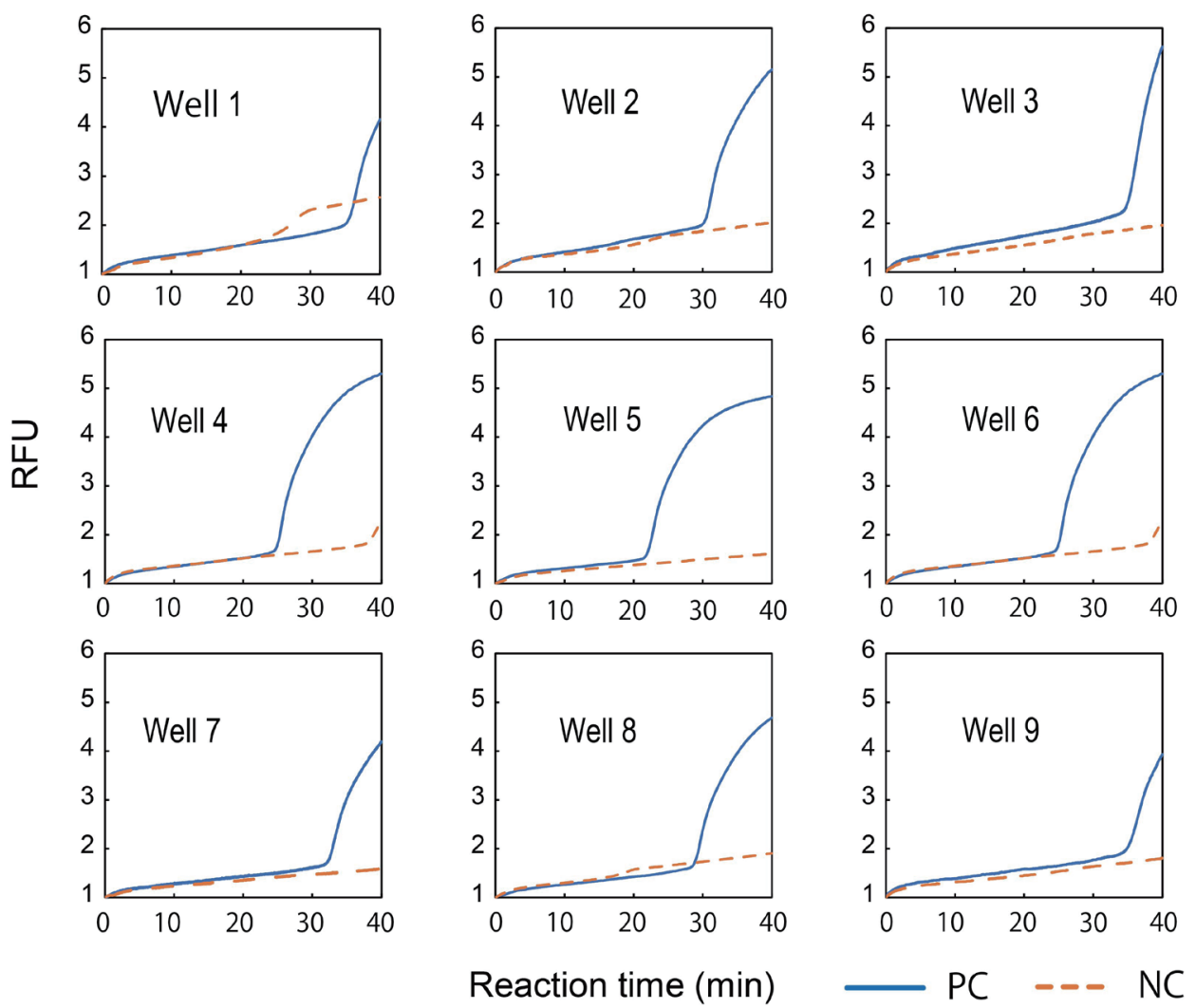

Fig. 7. (Color online) Time courses of the fluorescence intensity of the solution containing the positive or negative control in the nine microwells during the LAMP reaction.

Koshihikari rapidly increased from 17 to 30 min after the start of the LAMP reaction, whereas the fluorescence intensity of the solutions containing the other types of rice did not change markedly. On the other hand, when test reagent No. 2 was used, the fluorescence intensity of the three solutions containing Akitakomachi, Hitomebore, and Yumepirika markedly increased, whereas that of the solution containing Koshihikari hardly changed. A comparison of the fluorescence intensities obtained 40 min after starting the amplification is shown in Fig. 8(b). From the comparison of the fluorescence intensities of the sample solutions, the positive control, and the negative control, it was concluded that the LAMP reaction using Koshihikari was positive in test reagent No. 1 and negative in test reagent No.2. For the other samples, opposite results were obtained. These results agree with those in the manual ${ }^{(37)}$ and previous visual examination.

As described above, the microplate reader developed in this work was successfully used in the LAMP reaction using DNA extracted from real samples. The microplate reader will be useful for on-site gene testing in various fields since it is handheld and portable. 
(a)

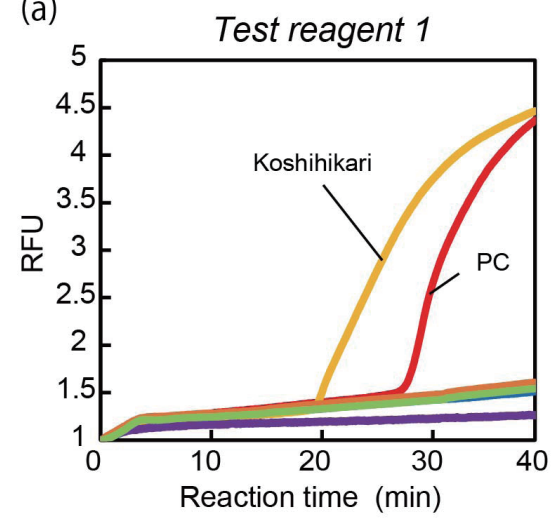

(b)

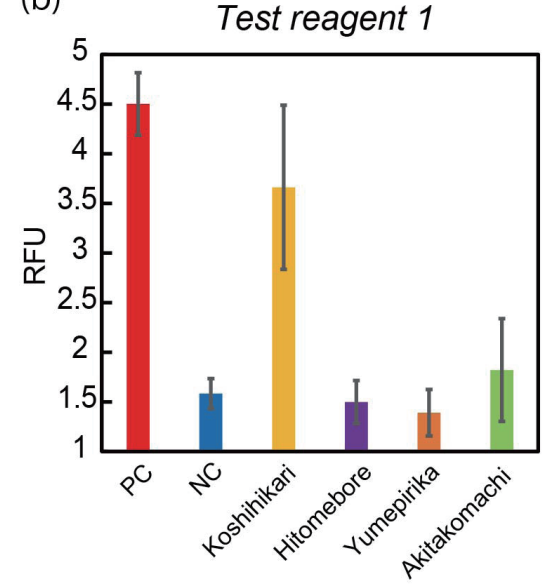

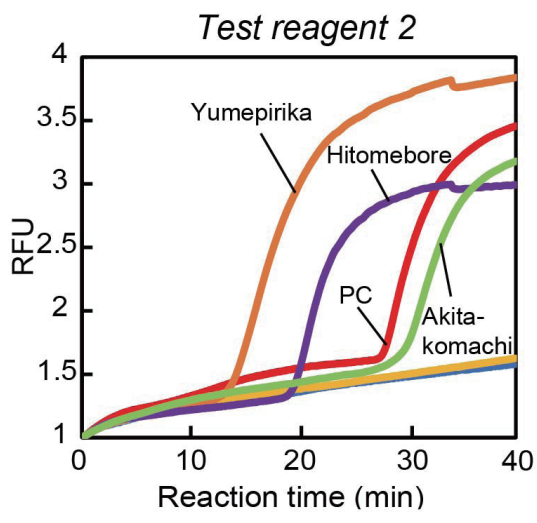

Test reagent 2

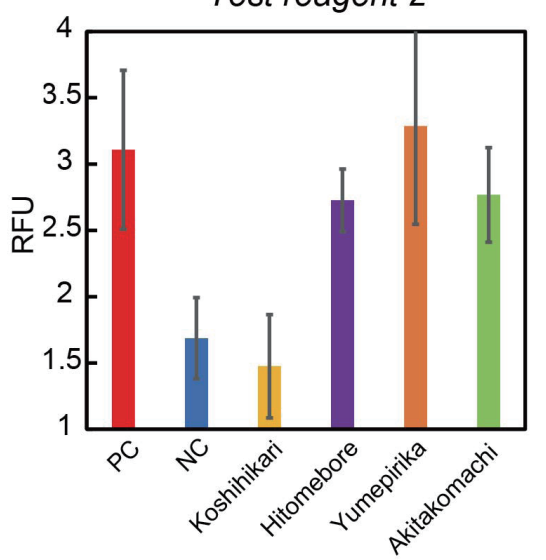

Fig. 8. (Color online) Results of the variety discrimination of rice. (a) Time courses of the fluorescence intensity of the sample solutions in the microwells during the LAMP reaction. (b) Comparison of the fluorescence intensities at 40 min.

\section{Conclusions}

In this study, a microplate reader equipped with a flat ITO glass heater with high optical transparency was developed and the simultaneous monitoring of the LAMP reaction based on fluorescence detection was demonstrated. The combination of the transparent ITO glass heater and the detection system using LEDs and PDs enabled the device configuration to be simplified and the analysis system to be miniaturized. This system is also suitable for other analysis methods (for example, other isothermal gene amplifications, hybridization, cell analysis, and measurements utilizing enzyme reactions).

In addition, the developed system is expected to be applied to the quantification of DNA. Using the present microplate reader, it is difficult to directly compare the fluorescence intensities in the different microwells because of small differences in the sensitivity of the PDs, the wavelengths and intensities of the LEDs, and the temperature in each microwell. However, the quantitation of DNA with the developed plate reader might be achieved in the future by adjusting the detection sensitivity and heating temperature at each well. Although the developed 
microplate reader was only used for the variety discrimination of rice in this study, it has the potential to be applied to various gene tests, such as infectious disease diagnosis, microorganism tests, and the detection of allergen substances. Since the microplate reader is handheld and can be operated using batteries, it is expected to be used in field tests when a disaster occurs.

\section{Acknowledgments}

This work was supported by Japan Society for the Promotion of Science (JSPS) KAKENHI (17K14504, 18H02009, 19H02751, 21H01968, 21H03578, 21K18985) and Tokyo Metropolitan Government.

\section{References}

1 O. Salamin, T. Kuuranne, M. Saugy, and N. Leuenberger: Drug Test. Anal. 9 (2017) 1731. https://doi. org/10.1002/dta.2324

2 T. Huang, L. Li, X. Liu, Q. Chen, X. Fang, J. Kong, M. S. Draz, and H. Cao: Anal. Methods 12 (2020) 5551. https://doi.org/10.1039/D0AY01768J

3 Y. Li, P. Fan, S. Zhou, and L. Zhang: Microb. Pathog. 107 (2017) 54. https://doi.org/10.1016/j.micpath.2017.03.016

4 F. Ahmad and S. A. Hashsham: Anal. Chim. Acta 733 (2012) 1. https://doi.org/10.1016/j.aca.2012.04.031

5 Q.-J. Zhou, L. Wang, J. Chen, R.-N. Wang, Y.-H. Shi, C.-H. Li, D.-M. Zhang, X.-J. Yan, and Y.-J. Zhang: J. Microbiol. Methods 104 (2014) 26. https://doi.org/10.1016/j.mimet.2014.06.008

6 S. Sul, M.-J. Kim, and H.-Y. Kim: Food Control 98 (2019) 194. https://doi.org/10.1016/j.foodcont.2018.11.025

7 E. Navarro, G. Serrano-Heras, M. J. Castaño, and J. Solera: Clin. Chim. Acta 439 (2015) 231. https://doi. org/10.1016/j.cca.2014.10.017

8 C. D. Ahrberg, B. R. Ilic, A. Manz, and P. Neužil: Lab Chip 16 (2016) 586. https://doi.org/10.1039/C5LC01415H

9 G. Mulberry, K. A. White, M. Vaidya, K. Sugaya, and B. N. Kim: PLoS ONE 12 (2017) 1. https://doi. org/10.1371/journal.pone.0179133

10 K. Shirato, N. Nao, S. Matsuyama, and T. Kageyama: Jpn. J. Infect. Dis. 73 (2020) 181. https://doi.org/10.7883/ yoken.JJID.2019.400

11 T. Notomi, H. Okayama, H. Masubuchi, T. Yonekawa, K. Watanabe, N. Amino, and T. Hase: Nucl. Acids Res. 28 (2000) e63. https://doi.org/10.1093/nar/28.12.e63

12 A. S. James and J. I. Alawneh: Diagnostics 10 (2020) 399. https://doi.org/10.3390/diagnostics10060399

13 A. K. Pantazis, G. Papadakis, K. Parasyris, A. Stavrinidis, and E. Gizeli: Sens. Actuators, B 319 (2020) 128161. https://doi.org/10.1016/j.snb.2020.128161

14 C. R. Phaneuf, B. Mangadu, H. M. Tran, Y. K. Light, A. Sinha, F. W. Charbonier, T. P. Eckles, A. K. Singh, and C.-Y. Koh: Biosens. Bioelectron. 120 (2018) 93. https://doi.org/10.1016/j.bios.2018.08.005

15 J. Fischbach, N. C. Xander, M. Frohme, and J. F. Glökler: BioTechniques. 58 (2015) 189. https://www.futurescience.com/doi/abs/10.2144/000114275

16 M. Safavieh, M. K. Kanakasabapathy, F. Tarlan, M. U. Ahmed, M. Zourob, W. Asghar, and H. Shafiee: ACS Biomater. Sci. Eng. 2 (2016) 278. https://doi.org/10.1021/acsbiomaterials.5b00449

17 M. Zhang, J. Ye, J. S. He, F. Zhang, J. F. Ping, C. Qian, and J. Wu: Anal. Chim. Acta 1099 (2020) 1. https://doi. org/10.1016/j.aca.2019.11.056

18 X. Fang, H. Chen, L. Xu, X. Jiang, W. Wu, and J. Kong: Lab Chip 12 (2012) 1495. https://doi.org/10.1039/ C2LC40055C

19 Y. Choi, Y. Song, Y. T. Kim, H. Kim, Y. M. Park, S. J. Lee, S. G. Im, and K. G. Lee: Sens. Actuators B 344 (2021) 130307. https://doi.org/10.1016/j.snb.2021.130307

20 F. W. Chow, T. T. Chan, A. R. Tam, S. Zhao, W. Yao, J. Fung, F. K. Cheng, G. C. Lo, S. Chu, K. L. Aw-Yong, J. Y. Tang, C. C. Tsang, H. K. Luk, A. C. Wong, K. S. Li, L. Zhu, Z. He, E. W. T. Tam, T. W. Chung, S. C. Y. Wong, T. L. Que, K. S. Fung, D. C. Lung, A. K. Wu, I. F. Hung, P. C. Woo, and S. K. Lau: Int. J. Mol. Sci. 21 (2020) 5380. https://doi.org/10.3390/ijms21155380

21 B. H. Park, S. J. Oh, J. H. Jung, G. Choi, J. H. Seo, D. H. Kim, E. Y. Lee, and T. S. Seo: Biosens. Bioelectron. 91 (2017) 334. https://doi.org/10.1016/j.bios.2016.11.063 
22 Y. Yao, N. Zhao, W. Jing, Q. Liu, H. Lu, W. Zhao, W. Zhao, Z. Yuan, H. Xia, and G. Sui: Sens. Actuators, B 333 (2021) 129521. https://doi.org/10.1016/j.snb.2021.129521

23 G. Xun, S. T. Lane, V. A. Petrov, B. E. Pepa, and H. Zhao: Nat. Commun. 12 (2021) 2905. https://doi. org/10.1038/s41467-021-23185-X

24 M.-Y. Yi, L. Ling, S. B. Neogi, Y.-S. Fan, D.-Y. Tang, S. Yamasaki, L. Shi, and L. Ye: Food Control 41 (2014) 91. https://doi.org/10.1016/j.foodcont.2014.01.005.

25 H. Q. Nguyen, V. D. Nguyen, H. V. Nguyen, and T. S. Seo: Sci. Rep. 10 (2020) 15123. https://doi.org/10.1038/ s41598-020-72095-3

26 X. Lin, X. Jin, W. Du, X. Shan, Q. Huang, R. Fu, W. Lv, H. Yang, Y. Su, and G. Huang: Anal. Methods 13 (2021) 3569. https://doi.org/10.1039/D1AY00953B

27 H. V. Nguyen, V. D. Nguyen, F. Liu, and T. S. Seo: ACS Omega 5 (2020) 22208. https://doi.org/10.1021/ acsomega.0c02317

28 A. H. Velders, C. Schoen, and V. Saggiomo: BMC Res. Notes 11 (2018) 93. https://doi.org/10.1186/s13104-018$\underline{3197-9}$

29 K. Yang, K. Cho, K. Im, and S. Kim: J. Inst. Korean Electr. Electron. Eng. 20 (2016) 171 (in Korean). https:// doi.org/10.7471/ikeee.2016.20.2.171

30 S. K. Jha, G.-S. Joo, G.-S. Ra, H. H. Lee, and Y.-S. Kim: IEEE Sens. J. 11 (2011) 2065. http://doi.org/10.1109/ JSEN.2011.2105262

31 K. Sun, A. Yamaguchi, Y. Ishida, S. Matsuo, and H. Misawa: Sens. Actuators, B 84 (2002) 283. https://doi. org/10.1016/S0925-4005(02)00016-3

32 S. R. Joung, J. Kim, Y. J. Choi, C. J. Kang, and Y. Kim: Proc. 2007 2nd IEEE Int. Conf. Nano/Micro Engineered and Molecular Systems (2007) 691. https://doi.org/10.1109/NEMS.2007.352113

33 C. W. Huang, H. Y. Chen, M.-H. Yen, Jeremy J. W. Chen, T.-H. Young, and J.-Y. Cheng: PLoS ONE 6 (2011) 1. https://doi.org/10.1371/journal.pone.0025928

34 C.-C. Chou and Y.-H. Huang: Sensors 12 (2012) 16660. https://doi.org/10.3390/s121216660

35 Z.-Y. Wu, K. Chen, B.-Y Qu, X.-X. Tian, X. J. Wang, and F. Fang: Anal. Chim. Acta 610 (2008) 89. https://doi. org/10.1016/j.aca.2007.12.044

36 H. Singh, K. Morioka, M. Shimojima, Van An L, H. Nakajima, A. Hemmi, K. Uchiyama, S. K. Loong, S. AbuBakar, M. Yang, and M. Sugamata: Jpn. J. Infect. Dis. 69 (2016) 435. https://doi.org/10.7883/yoken. JJID.2015.417

37 "Koshihikari” LAMP Identification Kit: https://genome.e-mp.jp/products/pdf/NE0121 Manual.pdf (accessed July 2021) 